\title{
Clinicopathological features of diabetic and nondiabetic renal diseases in type 2 diabetic patients with nephrotic-range proteinuria
}

\author{
Yu Ho Lee, MDa, Ki-Pyo Kim, MDa, Yang Gyun Kim, MD, PhDª, Ju-Young Moon, MD, PhDa, \\ Su Woong Jung, MDa, Eunji Park, $\mathrm{MD}^{\mathrm{a}}$, Jin Sug Kim, $\mathrm{MD}^{\mathrm{a}}$, Kyung-Hwan Jeong, MD, $\mathrm{PhD}^{\mathrm{a}}$, \\ Tae Won Lee, MD, PhDª, Chun-Gyoo Ihm, MD, PhDa , Young-ll Jo, MD, PhD ${ }^{\mathrm{b}}$, \\ Hoon-Young Choi, MD, PhD', Hyeong-Cheon Park, MD, PhD ${ }^{\mathrm{C}}$, So-Young Lee, MD, PhD', \\ Dong-Ho Yang, MD, PhD ${ }^{d}$, Joo-Hark Yi, MD, PhD ${ }^{e}$, Sang-Woong Han, MD, PhDe, Sang-Ho Lee, MD, PhDa,*
}

\begin{abstract}
Heavy proteinuria with or without features of nephrotic syndrome is associated with many primary and systemic diseases. For diabetic patients, distinguishing nondiabetic renal disease (NDRD) from diabetic nephropathy (DN) is important in choosing treatment modalities and determining renal prognosis. However, clinical relevance of heavy proteinuria is inconsistent with clinical DN assessments. This study investigated the clinicopathological features and renal outcomes of DN and NDRD in type 2 diabetic patients with nephrotic-range proteinuria.

We enrolled 220 cases of type 2 diabetic patients who underwent renal biopsy. They were grouped according to the presence of nephritic-range proteinuria and pathological features. Baseline characteristics, laboratory findings, types of pathological diagnosis, and renal outcomes were analyzed in patients with heavy proteinuria.

Upon kidney biopsy, 129 patients (58.6\%) showed nephritic-range proteinuria. Patients with heavy proteinuria (an average urine protein-to-creatinine ratio of $10,008 \pm 7307 \mathrm{mg} / \mathrm{gCr}$ ) showed lower serum albumin levels and higher total cholesterol levels, but did not show any difference in age, duration of diabetes, renal function, or the presence of retinopathy compared with those with mild-tomoderate proteinuria (an average urine protein-to-creatinine ratio of $1581 \pm 979 \mathrm{mg} / \mathrm{gCr}$ ). Renal biopsy revealed that the prevalence of NDRD was $37.2 \%$ in patients with heavy proteinuria, which was significantly lower than that in patients with mild-to-moderate proteinuria (63.7\%). The most common pathological types of NDRD were membranous nephropathy (41.7\%), IgA nephropathy (14.6\%), and minimal change disease (10.4\%). NDRD patients showed lower prevalence of diabetic retinopathy and better kidney function irrespective of proteinuria. Immunosuppressive treatment was administered more frequently in patients with heavy proteinuria (56.3\%) compared with patients with mild-to-moderate proteinuria (20\%) because of the pathological differences according to the amount of proteinuria. Renal outcomes were significantly worse in patients with DN than in patients with NDRD.

DN patients with heavy proteinuria exhibited different prevalence of NDRD and worse prognosis. Renal biopsy in type 2 diabetic patients should be more extensively considered to accurately diagnose NDRD, guide further management, and predict renal outcomes, especially in patients with nephrotic-range proteinuria.
\end{abstract}

Abbreviations: $\mathrm{NDRD}=$ nondiabetic renal disease, $\mathrm{DN}=$ diabetic nephropathy.

Keywords: diabetic nephropathy, nondiabetic renal disease, proteinuria, renal biopsy, type 2 diabetes

\footnotetext{
Editor: Weina Chen.

This research was supported by a grant from the National Research Foundation of Korea in 2012 (NRF-2012M3A9C6050511).

The authors have no conflicts of interest to disclose.

Supplemental Digital Content is available for this article.

${ }^{a}$ Division of Nephrology, Department of Internal Medicine, Kyung Hee University, ${ }^{b}$ Division of Nephrology, Department of Internal Medicine, Konkuk University Medical Center, ' Division of Nephrology, Department of Internal Medicine, Gangnam Severance Hospital, Yonsei University College of Medicine, Seoul, ${ }^{d}$ Division of Nephrology, Department of Internal Medicine, CHA Bundang Medical Center, CHA University, Seongnam, ${ }^{e}$ Division of Nephrology, Department of Internal Medicine, Hanyang University Guri Hospital, Guri, Korea.

* Correspondence: Sang-Ho Lee, Division of Nephrology, Department of Internal Medicine, Kyung Hee University Hospital at Gangdong, 892 Dongnam-ro, Gandonggu, Seoul, Korea (e-mail: Ishkidney@khu.ac.kr).
}

Copyright (c) 2017 the Author(s). Published by Wolters Kluwer Health, Inc.

This is an open access article distributed under the Creative Commons Attribution-NoDerivatives License 4.0, which allows for redistribution, commercial and noncommercial, as long as it is passed along unchanged and in whole, with credit to the author.

Medicine (2017) 96:36(e8047)

Received: 11 July 2017 / Received in final form: 17 August 2017 / Accepted: 21 August 2017

http://dx.doi.org/10.1097/MD.0000000000008047 


\section{Introduction}

Diabetic kidney disease, traditionally termed diabetic nephropathy $(\mathrm{DN})$, is the leading cause of end-stage renal disease (ESRD) worldwide. Despite major advances in medical care for diabetes mellitus (DM), the prevalence of DN among diabetic patients has not decreased over the last 3 decades. ${ }^{[1,2]}$ Moreover, the incidence of biopsy-proven DN is rapidly increasing, requiring further insight into its pathogenesis and novel therapeutic options. ${ }^{[3]}$

The natural course of DN has traditionally been described to be predictable; initial glomerular hyperperfusion is followed by microalbuminuria, overt proteinuria, and eventually progressive renal dysfunction. Accordingly, proteinuria, which is likely to be caused by $\mathrm{DN}$, is not an indication for renal biopsy, as pathological confirmation of DN rarely provides additional information regarding the management of patients. However, several studies have suggested that nondiabetic renal disease (NDRD) is common in diabetic patients, with the prevalence ranging from $27 \%$ to $79 \%$ among patients undergoing renal biopsy. ${ }^{[4-14]}$ Furthermore, recent studies have shown that the natural history of $\mathrm{DN}$ is substantially changing; the prevalence of proteinuria is decreasing, whereas the prevalence of reduced renal function without proteinuria is increasing. ${ }^{[1,15-17]}$ Thus, it is becoming increasingly important for clinicians to understand the pathophysiology of DN and to distinguish NDRD from DN among diabetic patients with chronic kidney disease.

Clinical predictors of NDRD in diabetic patients have been relatively well established across many studies. These include short duration of DM, absence of diabetic retinopathy, abrupt increase in serum creatinine, active urinary sediment, and the presence of hematuria. ${ }^{[4,5,7,9-14,18,19]}$ Given that the diagnostic spectrum of primary glomerular disease varies with proteinuria, it could be speculated that the types of renal disease and clinical features of proteinuric patients with DM might be different if they were classified according to the amount of proteinuria. However, proteinuria as a predictor of NDRD showed inconsistent results. The aims of this study were to identify clinical predictors of NDRD and to assess whether the pathological confirmation affects the choice of treatment modalities and prognosis in diabetic patients with nephroticrange proteinuria.

\section{Materials and methods}

\subsection{Patient selection and study design}

Upon reviewing medical records, we enrolled 220 patients with type 2 diabetes who underwent renal biopsy at 6 different medical centers (Kyung Hee University Hospital at Gangdong, Kyung Hee Medical Center, Konkuk University Medical Center, Gangnam Severance Hospital, CHA Bundang Medical Center, and Hanyang University Guri Hospital) from January 2012 to December 2016. Baseline characteristics and clinical parameters of enrolled patients according to hospitals are described in supplementary Table 1, http://links.lww.com/MD/B861. Indications for renal biopsy included overt proteinuria without diabetic retinopathy, short duration of $\mathrm{DM}(<5 \mathrm{y})$, the presence of active urinary sediment and/or hematuria, and unexplained decline in renal function. Patients with nephrotic syndrome, which was defined by nephrotic-range proteinuria $(\geq 3500 \mathrm{mg} / \mathrm{d}$ of proteinuria), pitting edema, hypoalbuminemia, and hypercholesterolemia, were also eligible for renal biopsy. Hypoalbuminemia was defined as levels of serum albumin $<3.5 \mathrm{~g} / \mathrm{dL}$, and hypercholesterolemia was defined as levels of serum total cholesterol $>260$ $\mathrm{mg} / \mathrm{dL}$ or current use of a statin. Patients with inadequate medical data, insufficient biopsy samples, or renal transplant recipients were excluded in this study.

Age, sex, height, weight, duration of DM, and the presence of hypertension were recorded, and routine laboratory analyses including HbA1c, hemoglobin, serum albumin, creatinine, and total cholesterol were also collected at the time of renal biopsy. Diabetic retinopathy was diagnosed by experienced ophthalmologists at each hospital. Urine was evaluated for the presence of hematuria and proteinuria. The presence of hematuria was defined by 5 or more red blood cells in 1 high-power field. The amount of proteinuria was measured by 24 -hour urine collection, if possible, or calculated as spot urine proteinto-creatinine ratio (PCR, $\mathrm{mg} / \mathrm{gCr}$ ). Serum creatinine was standardized with calibration traceable to an isotope-dilution mass spectrometry reference measurement procedure at each hospital, and the estimated glomerular filtration rate (eGFR) was calculated by the Modification of Diet in Renal Disease (MDRD) Study equation: $175 \times(\mathrm{Scr})^{-1.154} \times(\mathrm{Age})^{-0.203} \times$ (0.742, if female). The institutional review board (IRB) from each hospital approved this study.

\subsection{Pathological analyses and pathological classifications of enrolled patients}

All biopsy specimens were processed by standard methods for routine analyses by light microscopy, immunofluorescence, and electron microscopy, and were subsequently examined by expert pathologists at each hospital. Pathologic diagnosis of DN was made based on the following criteria ${ }^{[20]}$ : diffuse thickening of the glomerular basement membrane $(>395 \mathrm{~nm}$ in females and $>430$ $\mathrm{nm}$ in males) and mesangial expansion with or without nodular glomerulosclerosis, which all remained unexplained by other glomerular diseases.

Depending on the pathological findings, patients were divided into the following 3 groups: group I, isolated DN; group II, NDRD superimposed on DN; and group III, isolated NDRD.

\subsection{Follow-up and renal outcome}

Patients diagnosed with isolated DN received conservative management without immunosuppressive agents, unless other indications were present. Patients with NDRD superimposed on DN or isolated NDRD were treated according to the Kidney Disease Improving Global Outcomes (KDIGO) practical guidelines on glomerulonephritis. ${ }^{[21]}$ Patients visited outpatient clinics regularly for the assessment of renal function and proteinuria. Renal outcome was assessed by progression to ESRD.

\subsection{Statistical analysis}

All statistical analyses were performed with SPSS for Windows, version 20.0 (SPSS, Chicago, IL). Baseline clinical data are expressed as the mean \pm SD or as the numbers of patients and percentages. Independent $t$ tests, analysis of variance (ANOVA), and $\chi^{2}$ tests were used to compare baseline characteristics and laboratory findings, as appropriate. Multiple logistic regression analysis was used to assess the clinical predictors of NDRD and prognostic factors of renal outcomes. Finally, a time-to-event analysis for ESRD was performed with a $\log$-rank test. $P$ values $<.05$ were considered statistically significant. 
Table 1

Comparison of clinical features and laboratory findings between diabetic patients with nephrotic-range proteinuria and those with non-nephrotic-range proteinuria.

\begin{tabular}{lccr}
\hline & $\begin{array}{c}\text { Patients with } \\
\text { nephrotic-range } \\
\text { proteinuria (n=129) }\end{array}$ & $\begin{array}{c}\text { Patients with } \\
\text { non-nephrotic-range } \\
\text { proteinuria (n=91) }\end{array}$ & $\boldsymbol{P}$ \\
\hline Age, y & $57.6 \pm 12.3$ & $57.4 \pm 13.1$ & .921 \\
Sex (male, \%) & $75(58.1)$ & $50(54.9)$ & .638 \\
BMl, kg/m² & $25.2 \pm 4.1$ & $25.5 \pm 4.1$ & .685 \\
Duration of DM, y & $10.2 \pm 8.1$ & $8.9 \pm 7.2$ & .223 \\
Hypertension (n, \%) & $113(87.6)$ & $72(79.1)$ & .090 \\
Diabetic retinopathy (n, \%) & & & .201 \\
$\quad$ No & $61(47.3)$ & $51(56.0)$ & \\
$\quad$ Yes & $68(52.7)$ & $40(44.0)$ & \\
HbA1c, \% & $7.3 \pm 2.0$ & $7.3 \pm 1.5$ & .975 \\
Hemoglobin, g/dL & $11.7 \pm 9.4$ & $11.5 \pm 2.8$ & .908 \\
Creatinine, mg/dL & $2.27 \pm 1.83$ & $2.33 \pm 2.21$ & .864 \\
eGFR, mL/min & $46.8 \pm 34.9$ & $47.6 \pm 30.5$ & .914 \\
Albumin, g/dL & $2.93 \pm 0.67$ & $3.76 \pm 0.66$ & $<.001$ \\
Total cholesterol, mg/dL & $223.9 \pm 96.4$ & $163.8 \pm 51.5$ & $<.001$ \\
Urine PCR, mg/gCr & $10008 \pm 7307$ & $1581 \pm 979$ & $<.001$ \\
Hematuria (n, \%) & & & .189 \\
$\quad$ No & $65(50.4)$ & $54(59.3)$ & \\
$\quad$ Yes & $64(49.6)$ & $37(40.7)$ & \\
Pathologic diagnosis ${ }^{*}$ (n, \%) & & & $<.001$ \\
$\quad$ Group I & $81(62.8)$ & $33(36.3)$ & \\
Group II & $17(13.2)$ & $3(3.3)$ & \\
Group III & $31(24.0)$ & $55(60.4)$ & \\
\hline
\end{tabular}

Data are expressed as mean $\pm \mathrm{SD}$ or number of patients (percentage).

$\mathrm{BMI}=$ body mass index, $\mathrm{DM}=$ diabetes mellitus, eGFR $=$ estimated glomerular filtration rate, $\mathrm{HbA1C}=$ glycosylated hemoglobin, PCR = protein-to-creatinine ratio.

"Group I, isolated diabetic nephropathy; group II, nondiabetic renal disease superimposed on diabetic nephropathy; group III, isolated nondiabetic renal disease.

\section{Results}

\subsection{Baseline demographics and clinical characteristics of enrolled patients}

Table 1 summarizes the baseline demographics and clinical characteristics of the enrolled patients. We first divided patients according to the amount of proteinuria: patients with heavy proteinuria (urine PCR $>3500 \mathrm{mg} / \mathrm{gCr}, \mathrm{n}=129$ ) with an average urine PCR of $10,008 \mathrm{mg} / \mathrm{gCr}$, and those with mild-to-moderate proteinuria (urine PCR $<3500 \mathrm{mg} / \mathrm{gCr}, \mathrm{n}=91$ ) with an average urine PCR of $1581 \mathrm{mg} / \mathrm{gCr}$. The mean age, sex, body mass index, mean duration of diabetes, and the prevalence of diabetic retinopathy showed no difference between groups. However, serum albumin was significantly lower, and total cholesterol was higher in patients with heavy proteinuria compared with those with mild-to-moderate proteinuria $(2.93$ vs $3.76 \mathrm{~g} / \mathrm{dL}, P<.001$ and 223.9 vs $163.8 \mathrm{mg} / \mathrm{dL}, P<.001$, respectively). Renal function at presentation was similar, with mean eGFR of $46.8 \mathrm{~mL} / \mathrm{min}$ in patients with heavy proteinuria and $47.6 \mathrm{~mL} / \mathrm{min}$ in those with mild-to-moderate proteinuria. The prevalence of hematuria was also similar in both groups.

\subsection{The prevalence and clinical characteristics of NDRD among patients with DM}

Pathological analyses revealed that isolated DN (group I) was diagnosed in 114 patients ( 81 patients with heavy proteinuria and 33 patients with mild-to-moderate proteinuria) (Table 1). Twenty patients were diagnosed with NDRD superimposed on DN (group II) (17 patients with heavy proteinuria and 3 patients with mild-to-moderate proteinuria), and 86 patients were diagnosed with isolated NDRD (group III) (31 patients with heavy proteinuria and 55 patients with mild-to-moderate proteinuria). Overall, the prevalence of NDRD significantly differed according to the amount of proteinuria. Isolated $\mathrm{DN}$ was more common in patients with heavy proteinuria $(81 / 129,62.8 \%)$, whereas isolated NDRD was more common in patients with mild-tomoderate proteinuria $(55 / 91,60.4 \%)$. Pathological types of NDRD in groups II and III are listed in Table 2. Membranous nephropathy was the most commonly diagnosed NDRD among patients with heavy proteinuria. However, IgA nephropathy was the most common type of NDRD among patients with mild-tomoderate proteinuria. There was no case of crescentic $\operatorname{IgA}$ nephropathy, and all cases of crescentic glomerulonephritis were caused by antineutrophil cytoplasmic antibody (ANCA)-associated vasculitis.

Table 3 shows the clinical features and laboratory findings of patients according to the amount of proteinuria and pathological classifications. The mean duration of DM did not differ across

\section{Table 2}

Nondiabetic renal diseases among patients in group II and III.

\begin{tabular}{|c|c|c|c|c|c|c|}
\hline & \multicolumn{3}{|c|}{ Patients with nephrotic-range proteinuria $(n=48)$} & \multicolumn{3}{|c|}{ Patients with non-nephrotic-range proteinuria $(n=58)$} \\
\hline & Group $\|^{*}(n=17)$ & Group III $(n=31)$ & Total & Group II" $(n=3)$ & Group III* $(n=55)$ & Total \\
\hline Membranous nephropathy & 9 & 11 & 20 & 1 & 3 & 4 \\
\hline MCD & 1 & 4 & 5 & 0 & 1 & 1 \\
\hline Crescentic glomerulonephritis & 1 & 2 & 3 & 0 & 6 & 6 \\
\hline MPGN & 0 & 3 & 3 & 0 & 0 & 0 \\
\hline FSGS & 1 & 1 & 2 & 0 & 2 & 2 \\
\hline Lupus nephritis & 0 & 1 & 1 & 0 & 1 & 1 \\
\hline Acute interstitial nephritis & 0 & 0 & 0 & 0 & 7 & 7 \\
\hline Acute tubular necrosis & 0 & 0 & 0 & 1 & 5 & 6 \\
\hline Others ** & 1 & 3 & 4 & 1 & 10 & 11 \\
\hline
\end{tabular}

FSGS = focal segmental glomerulosclerosis, MCD = minimal change disease, MPGN= membranoproliferative glomerulonephritis, PIGN= post infectious glomerulonephritis

* Group II, nondiabetic renal disease superimposed on diabetic nephropathy; Group III, isolated nondiabetic renal disease.

${ }^{* *}$ Others include hypertensive nephrosclerosis, non-IgA mesangial proliferative glomerulonephritis, nonspecific focal effacement of foot process, and myeloma cast nephropathy. 
Clinical features and laboratory findings of patients according to the degree of proteinuria and pathologic classifications.

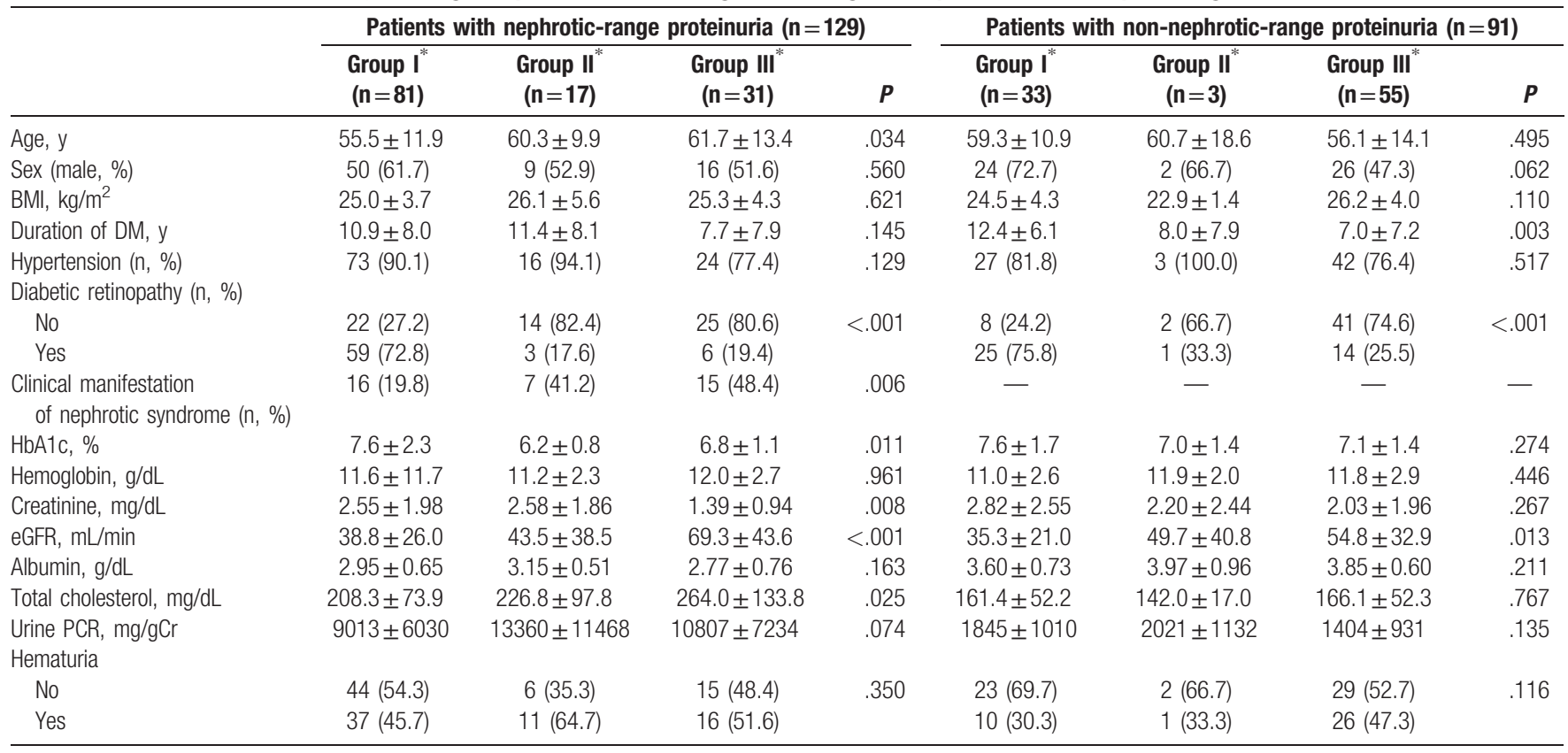

Data are expressed as mean $\pm S D$ or number of patients (percentage).

$\mathrm{BMI}=$ body mass index, $\mathrm{DM}=$ diabetes mellitus, eGFR = estimated glomerular filtration rate, $\mathrm{HbA1c}=$ glycosylated hemoglobin, $\mathrm{PCR}=$ protein-to-creatinine ratio.

* Group I, isolated diabetic nephropathy; group II, nondiabetic renal disease superimposed on diabetic nephropathy; group III, isolated nondiabetic renal disease.

groups in patients with heavy proteinuria (10.9 vs 11.4 vs $7.7 \mathrm{y}$, group I vs II vs III; $P=.145$ ). This is in contrast to the findings of patients with mild-to-moderate proteinuria, for which mean duration of DM in the isolated DN group was significantly longer than those in the NDRD superimposed on DN and isolated NDRD groups (12.4 vs 8.0 vs 7.0 y, group I vs II vs III; $P=.003$ ). Diabetic retinopathy was more frequently diagnosed in the isolated DN group $(72.8 \%$ vs $17.6 \%$ vs $19.4 \%$, group I vs II vs III; $P<.001$ in patients with heavy proteinuria and $75.8 \%$ vs $33.3 \%$ vs $25.5 \%$, group I vs II vs III; $P<.001$ in patients with mild-to-moderate proteinuria). eGFR was lower in patients with isolated DN regardless of the amount of proteinuria (38.8 vs 43.5 vs $69.3 \mathrm{~mL} / \mathrm{min}$, group I vs II vs III; $P<.001$ in patients with heavy proteinuria and 35.3 vs 49.7 vs $54.8 \mathrm{~mL} / \mathrm{min}$, group I vs II vs III; $P=.013$ in patients with mild-to-moderate proteinuria). Poor glycemic control (higher levels of HbA1c) was found in the isolated DN group, especially among patients with heavy proteinuria $(7.6 \%$ vs $6.2 \%$ vs $6.8 \%, P=0.011)$. Urine PCR and the prevalence of hematuria showed no difference across each pathological group. The prevalence of full-blown nephrotic syndrome in patients with heavy proteinuria was significantly lower in the isolated $\mathrm{DN}$ group $(19.8 \%$ vs $41.2 \%$ vs $48.4 \%$, group I vs II vs III; $P=0.006$ ).

\subsection{Clinical predictors of NDRD}

Clinical predictors of NDRD were assessed by multivariate logistic regression analysis, with all variables adjusted for age, sex, the presence of hypertension and eGFR (Table 4). Intriguingly, predictors of NDRD were different between patients with heavy proteinuria and those with mild-to-moderate proteinuria. A shorter duration of DM, which is a well-known clinical predictor of NDRD, was not associated with NDRD in patients with heavy proteinuria [odds ratio (OR) 0.961, confidence interval (CI) $0.910-1.015, P=0.153$ ]. By contrast, a significant correlation between short duration of DM and the probability of NDRD was observed in patients with mild-tomoderate proteinuria (OR 0.898, CI $0.840-0.960, P=.002$ ).

Table 4

Clinical predictors of nondiabetic renal diseases.

\begin{tabular}{|c|c|c|c|c|c|c|c|c|}
\hline & \multicolumn{4}{|c|}{ Patients with nephrotic-range proteinuria $(n=129)$} & \multicolumn{4}{|c|}{ Patients with nonnephrotic-range proteinuria $(n=91)$} \\
\hline & $\beta$-Estimate & Adjusted OR & 95\% CI & $P$ & $\beta$-Estimate & Adjusted OR & $95 \% \mathrm{Cl}$ & $P$ \\
\hline Age & 0.047 & 1.048 & $1.013-1.084$ & .006 & 0.019 & 1.020 & $0.972-1.069$ & .423 \\
\hline Duration of DM & -0.040 & 0.961 & $0.910-1.015$ & .153 & -0.108 & 0.898 & $0.840-0.960$ & .002 \\
\hline Absence of diabetic retinopathy & 2.730 & 15.33 & $5.211-45.121$ & $<.001$ & 2.694 & 14.79 & $3.520-62.112$ & $<.001$ \\
\hline $\mathrm{HbA1c}$ & -0.444 & 0.642 & $0.456-0.904$ & .011 & -0.280 & 0.756 & $0.531-1.075$ & .120 \\
\hline eGFR & 0.020 & 1.020 & $1.007-1.033$ & .002 & 0.036 & 1.037 & $1.014-1.059$ & .001 \\
\hline
\end{tabular}

Data shown are analyzed after adjustment with age, sex, the presence of hypertension, and eGFR.

$\mathrm{Cl}=$ confidence interval, $\mathrm{DM}=$ diabetes mellitus, eGFR $=$ estimated glomerular filtration rate, $\mathrm{HbA1c}=$ glycosylated hemoglobin, $\mathrm{OR}=$ odds ratio . 
Management and renal outcomes of patients.

\begin{tabular}{|c|c|c|c|c|c|c|c|}
\hline & \multicolumn{4}{|c|}{ Patients with nephrotic-range proteinuria $(n=129)$} & \multicolumn{3}{|c|}{ Patients with non-nephrotic-range proteinuria ${ }^{* * *}(\mathrm{n}=\mathbf{8 8})$} \\
\hline & $\begin{array}{c}\text { Group I } \\
(\mathrm{n}=\mathbf{8 1})\end{array}$ & $\begin{array}{l}\text { Group II } \\
(n=17)\end{array}$ & $\begin{array}{c}\text { Group III } \\
(\mathrm{n}=31)\end{array}$ & $P$ & $\begin{array}{l}\text { Group I* } \\
(n=33)\end{array}$ & $\begin{array}{c}\text { Group III } \\
(n=55)\end{array}$ & $\boldsymbol{P}$ \\
\hline Duration of follow up, mo & $20.2 \pm 13.5$ & $26.1 \pm 14.5$ & $19.0 \pm 12.6$ & .248 & $21.5 \pm 14.5$ & $23.9 \pm 16.0$ & .507 \\
\hline Use of immunosuppressant (n, \%) & $3(3.7)$ & $7(41.2)$ & $20(64.5)$ & $<.001$ & $0(0)$ & $11(20.0)$ & .006 \\
\hline Annual changes of eGFR, $\mathrm{mL} / \mathrm{min} / \mathrm{y}$ & $-10.41 \pm 11.42$ & $-5.62 \pm 12.71$ & $-4.35 \pm 14.33$ & .013 & $-4.5 \pm 6.3$ & $3.4 \pm 11.6$ & .003 \\
\hline Progression to ESRD** $(\mathrm{n}, \%)$ & $26 / 65(40.0)$ & $3 / 14(21.4)$ & $0 / 28(0)$ & $<.001$ & $10 / 30(33.3)$ & $1 / 41(2.4)$ & .004 \\
\hline
\end{tabular}

Data are expressed as mean $\pm S D$ or number of patients (percentage).

$\mathrm{ARB}=$ angiotensin receptor blocker, $\mathrm{ACE}=$ =angiotensin-converting enzyme inhibitor, eGFR=estimated glomerular filtration rate, ESRD =end-stage renal disease.

${ }^{*}$ Group I, isolated diabetic kidney disease; group II, nondiabetic renal disease superimposed on diabetic kidney disease; group III, isolated nondiabetic renal disease.

${ }^{* *}$ Patients with follow-up period of $<6$ months were excluded in this analysis.

**** Group II was excluded from the analysis because the number of patients was too small to be analyzed.

Older age was positively associated with NDRD in patients with heavy proteinuria, whereas this association was not found in those with mild-to-moderate proteinuria (OR 1.048, CI $1.013-1.084, P=.006$ and OR 1.020, CI 0.972-0.069, $P=.423$, respectively). The absence of diabetic retinopathy was a strong clinical predictor of NDRD, in accordance with the previous studies (OR 15.33, CI 5.211-45.121, $P<.001$ in patients with heavy proteinuria and OR 14.79 , CI 3.520-62.112, $P<0.001$ in patients with mild-to-moderate proteinuria). Less severe renal impairment was also an independent predictive factor of NDRD regardless of the amount of proteinuria (OR 1.020, CI $1.007-1.033, P=.002$ in patients with heavy proteinuria and OR 1.037 , CI $1.014-1.059, P=.001$ in patients with mild-tomoderate proteinuria).

\subsection{Treatment modality and renal outcomes of patients}

Further management and renal outcomes after kidney biopsy are summarized in Table 5. Among patients with mild-to-moderate proteinuria, group II was excluded from the analysis because the number of patients was too small to be analyzed. Two patients who were diagnosed with crescentic glomerulonephritis in group III died of severe infection during treatment and were not included in the outcome analysis.

Mean duration of follow-up and the use of angiotensinconverting enzyme inhibitor/angiotensin receptor blocker were not different among groups. Immunosuppressant, primarily to control NDRD, were more frequently prescribed in patients with heavy proteinuria $(3.7 \%$ vs $56.2 \%$, group I vs II + III; $P<.001$ in patients with heavy proteinuria and $0 \%$ vs $20.0 \%$, group I vs III; $P=.006$ in patients with mild-to-moderate proteinuria). Annual decline in renal function was more severe in patients with isolated $\mathrm{DN}$ than in other groups, both among patients with heavy proteinuria and mild-to-moderate proteinuria $(-10.41$ vs -5.62 vs $-4.35 \mathrm{~mL} / \mathrm{min} / \mathrm{y}$, group I vs II vs III; $P=.013$ and -4.5 vs $3.4 \mathrm{~mL} / \mathrm{min} / \mathrm{y}$, group I vs III; $P=.003$, respectively). The incidence of ESRD was also significantly higher in patients with isolated DN $(40.0 \%$ vs $21.4 \%$ vs $0 \%$, group I vs II vs III; $P<.001$ in patients with heavy proteinuria and $33.3 \%$ vs $2.4 \%$, group I vs III; $P=.004$ in patients with mild-to-moderate proteinuria; Table 5 and Fig. 1). Renal outcomes according to the etiology of renal disease were further analyzed (Supplementary Figure 1, http://links.lww.com/MD/ B861). The overall prognosis of patients with biopsy-proven DN was poor, with 5-year renal survival rate of around $40 \%$. Meanwhile, most patients with NDRD showed excellent renal outcomes irrespective of the types of NDRD. Finally, analysis of the prognostic factors of renal outcomes revealed that lower eGFR and the pathologic diagnosis of DN were significantly associated with the development of ESRD, regardless of proteinuria (Table 6). Higher HbA1c and severe proteinuria
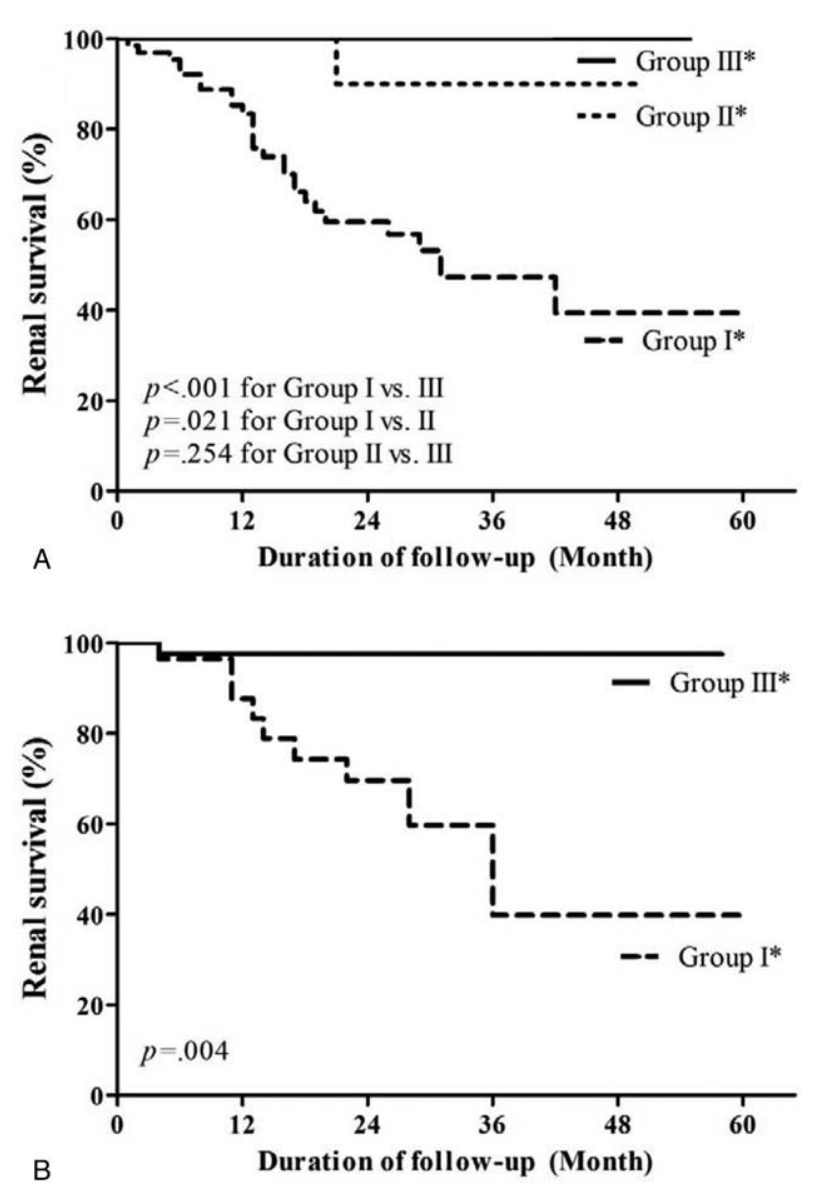

Figure 1. Renal outcomes of enrolled patients according to the degree of proteinuria and pathologic classification. (A) Renal survival in patients with nephrotic-range proteinuria, and $(B)$ renal survival in patients with nonnephrotic-range proteinuria. *Group I, isolated diabetic nephropathy; group II, nondiabetic renal disease superimposed on diabetic nephropathy; group III, isolated nondiabetic renal disease. ** Group II was excluded from the analysis because the number of patients was too small to be analyzed. 
Table 6

Risk factors of progression to end-stage renal diseases.

\begin{tabular}{|c|c|c|c|c|c|c|c|c|}
\hline & \multicolumn{4}{|c|}{ Patients with nephrotic-range proteinuria } & \multicolumn{4}{|c|}{ Patients with non-nephrotic-range proteinuria } \\
\hline & $\beta$-Estimate & Adjusted OR & $95 \% \mathrm{Cl}$ & $P$ & $\beta$-Estimate & Adjusted OR & $95 \% \mathrm{Cl}$ & $P$ \\
\hline Age & -0.017 & 0.983 & $0.935-1.032$ & .487 & 0.005 & 1.005 & $0.936-1.079$ & .885 \\
\hline Duration of DM & -0.032 & 0.968 & $0.899-1.043$ & .392 & 0.091 & 1.096 & $0.983-1.222$ & .100 \\
\hline $\mathrm{HbA1c}$ & 0.198 & 1.219 & $0.892-1.667$ & .214 & 0.669 & 1.951 & $1.132-3.363$ & .016 \\
\hline eGFR & -0.093 & 0.911 & $0.873-0.951$ & $<.001$ & -0.053 & 0.949 & $0.911-0.988$ & .011 \\
\hline Degree of proteinuria & 0.000 & 1.000 & $1.000-1.000$ & .388 & 0.001 & 1.001 & $1.000-1.002$ & .045 \\
\hline Presence of hematuria & 0.045 & 1.046 & $0.338-3.237$ & .938 & 0.000 & 1.000 & $0.204-4.906$ & 1.000 \\
\hline
\end{tabular}

$\mathrm{Cl}=$ confidence interval, $\mathrm{DM}=$ diabetes mellitus, $\mathrm{DN}=$ diabetic nephropathy, eGFR=estimated glomerular filtration rate, $\mathrm{HbA} 1 \mathrm{C}=$ glycosylated hemoglobin, NDRD = nondiabetic renal disease, $\mathrm{OR}=$ odds ratio.

were the risk factors of ESRD in patients with mild-to-moderate proteinuria, but not in those with heavy proteinuria.

\section{Discussion}

Numerous studies had been published regarding the prevalence, predictive factors, and clinical outcomes of NDRD in patients with DM, but the degree of proteinuria has not received significant attention to date. One possible explanation for this is that proteinuria does not show significant differences according to pathological classifications in most studies. Moreover, the amount of proteinuria varied greatly depending on the study design, ranging from 2000 to $8000 \mathrm{mg} / \mathrm{d}$, which might result from different policies, indications for renal biopsy and distinct prevalence of glomerulonephritis and DM according to ethnic diversities. Given that the underlying etiology of nephrotic- and non-nephrotic-range proteinuria differs significantly, we hypothesized that it would be reasonable to take a different approach by considering diabetic patients according to the degree of proteinuria. In this study, we demonstrated that the prevalence of NDRD among diabetic patients was significantly higher in patients with mild-to-moderate proteinuria than in patients with heavy proteinuria. On the contrary, isolated DN was diagnosed more frequently in patients presenting with heavy proteinuria. Furthermore, distinctive pathological types of coexisting NDRD were found between these 2 groups; membranous nephropathy was the most common type of NDRD in patients with heavy proteinuria, whereas IgA nephropathy was most commonly diagnosed in those with mild-to-moderate proteinuria. Taken together, our data suggest that assessing patients differently by the amount of proteinuria could yield additional information regarding the likelihood of NDRD and the necessity for renal biopsy.

A short duration of DM had been repeatedly shown to be a strong clinical predictor of NDRD in most studies. . $^{[4,5,7,9-14,18,19]}$ However, our results were partially discordant with previous studies. A short duration of DM was positively associated with NDRD in patients with mild-to-moderate proteinuria, whereas no association was found between the duration of DM and NDRD in patients with nephritic-range proteinuria. Furthermore, among the patients with isolated $\mathrm{DN}$, mean duration of DM was even shorter in patients with heavy proteinuria than in those with mild-to-moderate proteinuria, although a significant difference was not found ( $10.9 \mathrm{vs} 12.4 \mathrm{y}, P=.385)$. These findings are very intriguing, as they cannot be explained by the natural course of DN, which is characterized by a gradual increase in proteinuria over time. However, recent accumulating evidence has suggested that the traditional clinical course of DN is changing. Studies have suggested that the development of proteinuria and the decline in eGFR may not be sequential events but rather may have independent pathogeneses, and the widespread use of medications that block the renin angiotensin aldosterone system and improve glycemic control may be responsible for these phenomena. ${ }^{[22-25]}$ Large amounts of epidemiologic data have also revealed similar results, with a decreasing incidence of albuminuria in diabetic patients over the last decades. ${ }^{[1]}$ In our study, the prevalence of isolated DN was much higher in patients with heavy proteinuria than in those with mild-to-moderate proteinuria $(62.8 \%$ vs $36.3 \%, P<.001)$. However, as the natural course of DN continues to change, the prevalence of isolated $\mathrm{DN}$ in diabetic patients with heavy proteinuria will likely decrease, whereas the relative proportion of NDRD will increase. Accordingly, renal biopsy should be recommended more intensively in those patients.

Although numerous investigations had been performed to determine the predictive factors of NDRD, relatively few studies have compared the prognosis of diabetic patients with different pathological features. ${ }^{[4,5,13]}$ It is obvious that the prognosis of patients with isolated $\mathrm{DN}$ is significantly worse than that of other patients, with the risk of progression to ESRD ranging from $30 \%$ to $60 \%$ within 3 years after pathological diagnosis. ${ }^{[4,5,13]}$ On the contrary, it is evident that patients with isolated NDRD exhibit the best prognosis among diabetic patients, with $<10 \%$ of patients progressing to ESRD within 5 years after diagnosis. However, the prognosis of patients with NDRD superimposed on DN has shown conflicting results. Chang et al and Wong et al demonstrated that the prognoses of those patients were similar to the prognoses of patients with isolated $\mathrm{DN},{ }^{[5,13]}$ whereas our data showed that most of those patients maintained their renal function throughout the study period. It was difficult to explain the reasons for this discrepancy, as we could not obtain information regarding NDRD treatment or changes in clinical parameters over the course of follow-up in the 2 previous studies. Further large studies are mandatory to confirm the prognosis of diabetic patients with combined DN and NDRD.

Specific types of NDRD and DN may share some identical pathological features, which makes accurate diagnosis complicated. For example, patients with DN frequently show diffuse effacement of foot processes, which is also the only pathological feature of minimal change disease (MCD). ${ }^{[26]}$ In this case, it may be difficult for clinicians to distinguish isolated DN from MCD superimposed on $\mathrm{DN}$, unless obvious clinical manifestation 
favors MCD such as abrupt onset of proteinuria and nephrotic syndrome. Furthermore, early and/or atypical features of DN may be missed in patients who are diagnosed with isolated NDRD, as renal biopsy only provides a snapshot of the restricted area within the entire kidney. Accordingly, disease-specific markers for DN and other glomerular diseases are needed to overcome this problem.

Several limitations of our study should be mentioned. Currently, selection bias is an inevitable issue because renal biopsy is not routinely performed in patients with a high probability of having DN. Previous retrospective studies, which applied similar indications for renal biopsy, had also demonstrated that the prevalence of NDRD in diabetic patients was ranging from $27 \%$ to $79 \%$, similar to our study. ${ }^{[4-14]}$ On the contrary, our results showed that the prevalence of isolated DN was higher in patients with nephritic-range proteinuria. The prevalence of NDRD in patients with heavy proteinuria has been rarely reported. Some clinicians try to undergo renal biopsy to exclude several primary causes of nephrotic syndrome when diabetic patients show clear clinical manifestations of nephrotic syndrome. However, most diabetic patients with heavy proteinuria tend to be excluded from renal biopsy because "classical DN" usually implies heavy proteinuria. Taken together, we concluded that the prevalence of isolated $\mathrm{DN}$ would be more frequent in patients with nephritic-range proteinuria despite considerable selection bias based on indication for renal biopsy among diabetic patients with heavy proteinuria.

Another limitation is that we could not evaluate more detailed pathological subgroups, especially with regard to DN. The current pathological classification of DN was developed by the Renal Pathology Society, ${ }^{[20]}$ and the clinical relevance of this classification was evaluated in patients with isolated DN. ${ }^{[27]}$

In conclusion, diabetic patients with nephrotic-range proteinuria showed a distinctive pattern of clinical parameters as well as different prevalence of NDRD and clinical predictors of NDRD. Pathological diagnosis was valuable in predicting renal outcomes and choosing treatment modalities in patients with heavy and mild-to-moderate proteinuria. Recent updates on the natural course of DN will also change our perception and clinical approach to NDRD, and it is reasonable to more extensively consider renal biopsy in diabetic patients presenting with heavy proteinuria.

\section{References}

[1] Afkarian M, Zelnick LR, Hall YN, et al. Clinical manifestations of kidney disease among US adults with diabetes, 1988-2014. JAMA 2016;316:602-10.

[2] de Boer IH, Rue TC, Hall YN, et al. Temporal trends in the prevalence of diabetic kidney disease in the United States. JAMA 2011;305:2532-9.

[3] O'Shaughnessy MM, Hogan SL, Poulton CJ, et al. Temporal and demographic trends in glomerular disease epidemiology in the Southeastern United States, 1986-2015. Clin J Am Soc Nephrol 2017;12:614-23.

[4] Byun JM, Lee CH, Lee SR, et al. Renal outcomes and clinical course of nondiabetic renal diseases in patients with type 2 diabetes. Korean J Intern Med 2013;28:565-72.

[5] Chang TI, Park JT, Kim JK, et al. Renal outcomes in patients with type 2 diabetes with or without coexisting non-diabetic renal disease. Diabetes Res Clin Pract 2011;92:198-204.
[6] Chong YB, Keng TC, Tan LP, et al. Clinical predictors of non-diabetic renal disease and role of renal biopsy in diabetic patients with renal involvement: a single centre review. Ren Fail 2012;34:323-8.

[7] Lee EY, Chung CH, Choi SO. Non-diabetic renal disease in patients with non-insulin dependent diabetes mellitus. Yonsei Med J 1999;40: $321-6$.

[8] Mazzucco G, Bertani T, Fortunato M, et al. Different patterns of renal damage in type 2 diabetes mellitus: a multicentric study on 393 biopsies. Am J Kidney Dis 2002;39:713-20.

[9] Sharma SG, Bomback AS, Radhakrishnan J, et al. The modern spectrum of renal biopsy findings in patients with diabetes. Clin J Am Soc Nephrol 2013;8:1718-24.

[10] Soleymanian T, Hamid G, Arefi M, et al. Non-diabetic renal disease with or without diabetic nephropathy in type 2 diabetes: clinical predictors and outcome. Ren Fail 2015;37:572-5.

[11] Soni SS, Gowrishankar S, Kishan AG, et al. Non diabetic renal disease in type 2 diabetes mellitus. Nephrology (Carlton) 2006;11:533-7.

[12] Wilfred DC, Mysorekar VV, Venkataramana RS, et al. Nondiabetic renal disease in type 2 diabetes mellitus patients: a clinicopathological study. J Lab Physicians 2013;5:94-9.

[13] Wong TY, Choi PC, Szeto CC, et al. Renal outcome in type 2 diabetic patients with or without coexisting nondiabetic nephropathies. Diabetes Care 2002;25:900-5.

[14] Zhou J, Chen X, Xie Y, et al. A differential diagnostic model of diabetic nephropathy and non-diabetic renal diseases. Nephrol Dial Transplant 2008;23:1940-5.

[15] Kramer HJ, Nguyen QD, Curhan G, et al. Renal insufficiency in the absence of albuminuria and retinopathy among adults with type 2 diabetes mellitus. JAMA 2003;289:3273-7.

[16] Thomas MC, Macisaac RJ, Jerums G, et al. Nonalbuminuric renal impairment in type 2 diabetic patients and in the general population (national evaluation of the frequency of renal impairment cOexisting with NIDDM [NEFRON] 11). Diabetes Care 2009;32: 1497-502.

[17] Molitch ME, Steffes M, Sun W, et al. Development and progression of renal insufficiency with and without albuminuria in adults with type 1 diabetes in the diabetes control and complications trial and the epidemiology of diabetes interventions and complications study. Diabetes Care 2010;33:1536-43.

[18] Lin YL, Peng SJ, Ferng SH, et al. Clinical indicators which necessitate renal biopsy in type 2 diabetes mellitus patients with renal disease. Int J Clin Pract 2009;63:1167-76.

[19] Liu MY, Chen XM, Sun XF, et al. Validation of a differential diagnostic model of diabetic nephropathy and non-diabetic renal diseases and the establishment of a new diagnostic model. J Diabetes 2014;6:519-26.

[20] Tervaert TW, Mooyaart AL, Amann K, et al. Pathologic classification of diabetic nephropathy. J Am Soc Nephrol 2010;21:556-63.

[21] Radhakrishnan J, Cattran DC. The KDIGO practice guideline on glomerulonephritis: reading between the (guide)lines—application to the individual patient. Kidney Int 2012;82:840-56.

[22] Retnakaran R, Cull CA, Thorne KI, et al. Group USRisk factors for renal dysfunction in type 2 diabetes: U.K. Prospective Diabetes Study 74. Diabetes 2006;55:1832-9.

[23] Yokoyama H, Sone H, Oishi M, et al. Prevalence of albuminuria and renal insufficiency and associated clinical factors in type 2 diabetes: the Japan Diabetes Clinical Data Management study (JDDM15). Nephrol Dial Transplant 2009;24:1212-9.

[24] Afghahi H, Cederholm J, Eliasson B, et al. Risk factors for the development of albuminuria and renal impairment in type 2 diabetesthe Swedish National Diabetes Register (NDR). Nephrol Dial Transplant 2011;26:1236-43.

[25] Pugliese G. Updating the natural history of diabetic nephropathy. Acta Diabetol 2014;51:905-15.

[26] Alsaad KO, Herzenberg AM. Distinguishing diabetic nephropathy from other causes of glomerulosclerosis: an update. J Clin Pathol 2007;60: $18-26$.

[27] An Y, Xu F, Le W, et al. Renal histologic changes and the outcome in patients with diabetic nephropathy. Nephrol Dial Transplant 2015;30: 257-66. 